Editorials

\title{
Gc Protein-Derived Macrophage Activating Factor (GcMAF) and Autism: Do Clinical Results Require a Novel Interpretation?
}

\author{
Marco Ruggiero \\ dr.reinwald healthcare, Friedrich-Luber Street, 29·D-90592 Schwarzenbruck, Germany
}

Article history

Received: $14-09-2016$

Revised: $10-10-2016$

Accepted: 13-10-2016

Email: marco.drruggiero@gmail.com

\section{Introduction}

The Gc protein-derived Macrophage Activating Factor (GcMAF) has been the object of intense investigation since its first description (Yamamoto and Kumashiro, 1993) because of its potential therapeutic use in a number of different conditions associated with dysfunctions of the immune system. Interestingly, such an intense investigation has been paralleled by an equally intense controversy (Ugarte et al., 2014).

According to the hypothesis first proposed by Yamamoto and Kumashiro (1993), GcMAF derives from a plasma protein, the Gc protein (also known as vitamin D-binding protein), following the enzymatic removal of two sugars attached to threonine 420 during immune responses. The enzymes beta-galactosidase, released by activated B lymphocytes and sialidase, released by activated $\mathrm{T}$ lymphocytes, would remove galactose and sialic acid, thus exposing $\mathrm{N}$-acetylgalactosamine (GalNAc) as the remaining sugar moiety attached to threonine 420 of the Gc protein. GalNAc would then be the active site of GcMAF.

According to the hypothesis by Yamamoto et al., (1995), patients infected with HIV, cancer patients (Yamamoto et al., 1996), or patients with Lupus (Yamamoto et al., 1997), had elevated plasma levels of an enzyme, alpha-N-acetylgalactosaminidase (nagalase). This enzyme that would remove GalNAc from the Gc protein, thus preventing its conversion to the active GcMAF. Thus, according to this hypothesis, elevated nagalase levels would correspond to decreased production of endogenous GcMAF and, hence, to immunodeficiency (Yamamoto et al., 1996; 2008).

Based on this hypothesis, Yamamoto et al. proposed to produce GcMAF in the laboratory by enzymatically treating Gc protein extracted from human blood and to administer this GcMAF to patients with elevated nagalase in order to overcome the supposed deficiency of endogenous GcMAF. Such an administration would have restored the functionality of the immune system in HIV and cancer patients thus leading to "eradication" of HIV and cancer.

However, the papers where the results achieved by administering GcMAF produced in the laboratory to HIV patients or patients affected by breast or colon cancer, have been retracted and the entire rationale for such an approach has been severely criticized (Retraction, 2014a; 2014b; 2014c; Ugarte et al., 2014).

As a matter of fact, clinical observations and experimental findings demonstrate that the hypothesis on which GcMAF administration was based shows significant inconsistencies:

1. Subjects who cannot produce even a single molecule of GcMAF show no signs of immunodeficiency or predisposition to cancer; on the contrary, they have 
reduced risk of cancer. This observation refers to subjects who harbor the Gc2 allele only (Gc2 homozygotes) of the Gc protein. These individuals are unable to glycosylate the $\mathrm{Gc}$ protein on threonine 420 due to its substitution by lysine. Thus, there is no GalNAc in position 420. In other words, Gc2 homozygotes are unable to produce one single molecule of GcMAF. However, despite this fact, the risk of cancer in these individuals is decreased rather than increased as one would have expected given the absence of bona fide GcMAF (Abbas et al., 2008)

2. Subjects with elevated nagalase, such as autistic children, do not show signs of immunodeficiency (Bradstreet et al., 2012)

3. Subjects with cancer do not show decreased levels of endogenous GcMAF; on the contrary the level of their endogenous GcMAF not only is not different from healthy subjects, but it is significantly higher than the trace amounts of the exogenously administered GcMAF that should "eradicate" their cancer (Rehder et al., 2009)

4. Nagalase degrades GcMAF by removing GalNAc, thus rendering it inactive (Mohamad et al., 2002; Borges and Rehder, 2016). Therefore, in patients with elevated nagalase, the trace amounts of exogenously administered GcMAF would be immediately degraded

5. The procedure to produce GcMAF in the laboratory using the enzyme beta-galactosidase from E. Coli (Kuchiike et al., 2013; Uto et al., 2015) is not consistent with experimental data. Thus, this enzyme does not cleave galactose from the $\mathrm{Gc}$ protein (Borges and Rehder, 2016). Therefore, it is questionable whether using such a procedure even a single molecule of GcMAF is produced.

It is worth noticing that, despite these obvious inconsistencies, GcMAF has been independently studied for about two decades by several research groups that reported consistent results in vitro and in vivo (for a recent review, see Ruggiero et al., 2016). My former research group, among others, has witnessed the effects of GcMAF derived from human blood on human mononuclear cells and on angiogenesis (Pacini et al., 2010; 2012a), on human breast cancer cells (Pacini et al., 2012b; Thyer et al., 2013a) and on human neurons and glial cells (Morucci et al., 2015; Branca et al., 2015). We have also observed the clinical effects of GcMAF administration in the context of an integrated immunotherapeutic protocol for cancer (Ruggiero et al., 2014) and members of my former research group have described the effects of integrated GcMAF immunotherapy in diseases as diverse as cancer (Thyer et al., 2013b), chronic fatigue syndrome, multiple sclerosis, Lyme disease, amyotrophic lateral sclerosis, syphilis and autism (Thyer et al., 2013c).

These observations of ours have been independently corroborated, among others, by researchers from Japan who have reported encouraging results obtained using a similar immunotherapeutic approach in cancer (Inui et al., 2013; 2014; Inui et al., 2016a), multiple sclerosis (Inui et al., 2016b), serious infections and chronic fatigue syndrome (Inui et al., 2015).

In an effort to reconcile the results observed by us and many others with the inconsistencies described above, we recently hypothesized that the biological and clinical effects attributed to GcMAF are indeed to be ascribed to a glycosaminoglycan, chondroitin sulfate, that binds to Gc protein and GcMAF in plasma and other bodily fluids as well as on the surface of the cells of the immune system (Ruggiero et al., 2016). According to this hypothesis, the glycosylation status of the Gc protein is irrelevant in determining the biological and clinical effects that we and others have observed with GcMAF and the active GalNAc would the one that is a constituent part of chondroitin sulfate.

This novel hypothesis is particularly apt at explaining the results obtained with GcMAF in autism spectrum disorders as they were firstly described by the late Dr. Bradstreet (Bradstreet et al., 2012) and later confirmed by members of my former research group (Thyer et al., 2013c).

In the study by Bradstreet et al. (2012), it was observed that, in a cohort of forty autistic children, all subjects had elevated levels of nagalase. After an average 14 weeks of weekly subcutaneous injections with human blood-derived GcMAF, all autistic subjects, but one, showed a decrease of nagalase levels and a significant percentage of them showed improvement of the clinical symptoms of autism evaluated according to Clinical Global Impression of Improvement scale. These results are now difficult to interpret at the light of the recent observation that elevated nagalase should have degraded the exogenously administered GcMAF as it was demonstrated by Borges and Rehder (2016). The source of elevated nagalase could not be accounted for and the conundrum of the lack of signs of immunodeficiency in spite of elevated nagalase could not be explained.

These inconsistencies between the biochemistry of GcMAF and the clinical results appear all the more striking considering that GcMAF has been described by the late Dr. Bradstreet as "one of the most powerful tools I have ever used for autism" during his last public speech at the AutismOne Conference in May 2015 (the speech of Dr. Bradstreet is publicly available at https://www.youtube.com/watch? $\mathrm{v}=6 \mathrm{I} 2 \mathrm{Wr} 9 \mathrm{ihvV0}$ and his last words on GcMAF are at minute 52:26). 
If, however, we accept the hypothesis that the biological effects of GcMAF are either due to, or mediated by chondroitin sulfate, then all the inconsistencies cease to exist (Ruggiero et al., 2016). Thus, chondroitin sulfate is constituted by GalNAc, the supposed active site of GcMAF, but it is not affected by nagalase since this enzyme is not endowed with endoglycosidic function (Borges and Rehder, 2016).

Interestingly, chondroitin sulfate exhibits most, if not all, the biological and clinical properties attributed to GcMAF, including the anti-inflammatory and immune modulating effects recently reported by Theoharides et al. (2016) when referring to the use of GcMAF in autism. Thus, it is well known that glycosaminoglycans such as chondroitin sulfate are associated with central nervous system development, maintenance and disorders. The relevance of chondroitin sulfate in the pathogenesis of autism and other neurological and psychiatric disorders is corroborated by the evidence that, as a component of the extracellular matrix that interacts with the perineuronal nets, chondroitin sulfate plays a fundamental role in regulating synaptic functions and plasticity (Pantazopoulos and Berretta, 2016).

Furthermore, disaccharides derived from chondroitin sulfate have been implicated in the inhibition of neurodegeneration by influencing microglia activation (Ebert et al., 2008) in a manner consistent with what we had observed treating microglial cells in vitro with GcMAF (Branca et al., 2015). Consistent with these evidences, experimental and clinical data suggest that chondroitin sulfate might be a useful therapeutic agent in neurological diseases that are characterized by inflammation such as Parkinson's and Alzheimer's diseases, multiple sclerosis and amyotrophic lateral sclerosis (Vallières and du Souich, 2010) that are some of the conditions successfully treated with GcMAF (Thyer et al., 2013c).

Also the molecular structure of chondroitin sulfate is consistent with the hypothesis that this glycosaminoglycan is the molecule responsible for the macrophage stimulating activity that we and others had attributed to GcMAF. Thus, researchers at the Arizona State University, demonstrated that the macrophage stimulating activity resides in a GalNAc residue exposed in the context of an alpha helix (Bogani et al., 2006) and chondroitin sulfate is precisely composed of a chain of alternating GalNAc and glucuronic acid that are arranged in a right handed helical structure (Németh-Csóka et al., 1975). Therefore, it can be hypothesized that the GalNAc of chondroitin sulfate and not that exposed on the Gc protein is responsible for activating macrophages and directing the immune response (Wrenshall et al., 1999). This observation would explain the reason why Gc2 homozygotes, who cannot produce a single molecule of GcMAF but have plenty of chondroitin sulfate, do not suffer of immunodeficiency and are not predisposed to cancer (Abbas et al., 2008).

Furthermore, it is important to notice that the GcMAF used in the study on autism quoted above was obtained by enzymatic treatment of a plasma protein, the Gc protein, that had been isolated from human plasma by vitamin $\mathrm{D}_{3}$-Sepharose or actin-agarose affinity chromatography with no description of further purification steps aimed at removing plasma glycosaminoglycans (Bradstreet et al., 2012). It is well known that Gc protein belongs to the superfamily of albumin and it is present in fraction IV of Cohn-Oncley fractionation of human plasma. Since 2007, we had demonstrated that chondroitin sulfate is highly represented in this very Cohn-Oncley fraction (Cecchi et al., 2007) and, considering that in 1999, DiMartino and Kew had demonstrated that $\mathrm{Gc}$ protein binds to chondroitin sulfate (DiMartino and Kew, 1999), it can be deducted that Gc protein and hence the studied GcMAF, were associated and co-extracted with chondroitin sulfate.

Another point that requires reinterpretation is the role of nagalase in autism; thus, it is difficult to envisage the source of nagalase in those patients since autism is not associated with viruses known to produce the enzyme such as influenza virus (Yamamoto and Urade, 2005) or HIV-1 (Yamamoto, 2006). If, however, we consider that autism is associated with widespread inflammation and with higher concentration of proinflammatory cytokines (Masi et al., 2015), then it can be speculated that nagalase, being a lysosomal enzyme (Suh et al., 2015), may be interpreted as a marker of chronic inflammation rather than a marker of immunodeficiency. Since chondroitin sulfate is endowed with anti-inflammatory properties and it exerts this action upstream of the inflammasome by inhibiting activation of NF-kappaB transcription factors (Stabler et al., 2016), it is conceivable that the observed reduction of nagalase was due to chondroitin sulfate rather than to GcMAF.

\section{Conclusion}

The two most recent publications on GcMAF (Borges and Rehder, 2016; Ruggiero et al., 2016) help reinterpreting the biological and clinical results independently observed in vitro and in vivo by a number of researchers. The hypothesis that chondroitin sulfate may be responsible for the effects thus far attributed to GcMAF, solves al the inconsistencies and contradictions that have characterized this field of immunotherapy. Furthermore, this hypothesis lays the foundation for the development of non-proteinic macrophage activating factors that are not extracted from human blood, thus avoiding all the risks associated with human blood-derived products. 


\section{Author's Contribution}

Marco Ruggiero wrote and revised the manuscript.

\section{Conflict of Interest}

The Author is a retired full professor of molecular biology at the University of Firenze, Italy and he is currently a consultant for the company "dr. reinwald healthcare", a company that organizes seminars and trainings for therapists and commercializes nutritional supplements and therapy devices. None of the products or devices distributed by the company is mentioned in this article and the Author has not received financial compensation for writing this article. The Author is member of the Editorial Board of The American Journal of Immunology and is waived from the Article Processing fee for this contribution; the Author receives no remuneration for his editorial work.

\section{References}

[No authors listed], 2014a. Retraction of Immunotheraphy of metastatic breast cancer patients with vitamin D-binding protein-derived activating factor (GcMAF) by Yamamoto N., Suyama H., N. Yamamoto and N. Ushijima. Int. J. Cancer, 135: 1509-1509.

[No authors listed], 2014b. Retraction of Immunotherapy of metastatic colorectal cancer with vitamin Dbinding protein-derived macrophage-activating factor, GcMAF by Yamamoto, N., H. Suyama, H. Nakazato, N. Yamamoto and Y. Koga. Cancer Immunol. Immunother., 63: 1349-1349.

[No authors listed], 2014c. Retraction of Immunotherapy of HIV-infected patients with Gc protein-derived macrophage activating factor by Yamamoto N, N. Ushijima and Y. Koga. J. Med. Virol., 86: 1998-1998.

Abbas, S., J. Linseisen, T. Slanger, S. Kropp and E.J. Mutschelknauss et al., 2008. The Gc2 allele of the vitamin $\mathrm{D}$ binding protein is associated with a decreased postmenopausal breast cancer risk, independent of the vitamin D status. Cancer Epidemiol. Biomarkers Prev., 17: 1339-1343. PMID: 18559548

Bogani, F., E. McConnell, L. Joshi, Y. Chang and G. Ghirlanda, 2006. A designed glycoprotein analogue of Gc-MAF exhibits native-like phagocytic activity. J. Am. Chem. Soc., 128: 7142-7143. DOI: $10.1021 /$ ja0604212

Borges, C.R. and D.S. Rehder, 2016. Glycan structure of Gc Protein-derived Macrophage Activating Factor as revealed by mass spectrometry. Arch. Biochem. Biophys., 606: 167-179.

DOI: $10.1016 /$ j.abb.2016.08.006
Bradstreet, J.J., E. Vogelaar and L. Thyer, 2012. Initial observations of elevated alpha-Nacetylgalactosaminidase activity associated with autism and observed reductions from Gc proteinmacropahe activating factor injections. Autism Insights, 4: 31-38. DOI: 10.4137/AUI.S10485

Branca, J.J.V., G. Morucci, F. Malentacchi, S. Gelmini and M. Ruggiero et al., 2015. Effects of oxaliplatin and oleic acid Gc-protein-derived macrophageactivating factor on murine and human microglia. J. Neurosci. Res., 93: 1364-1377.

DOI: $10.1002 /$ jnr.23588

Cecchi, F., M. Ruggiero, R. Cappelletti, F. Lanini and S. Vannucchi, 2007. Improved method for analysis of glycosaminoglycans in glycosaminoglycan/protein mixtures: Application in Cohn-Oncley fractions of human plasma. Clin. Chim. Acta, 376: 142-149. DOI: $10.1016 /$ j.cca.2006.08.011

DiMartino, S.J. and R.R. Kew, 1999. Initial characterization of the vitamin D binding protein (Gc-globulin) binding site on the neutrophil plasma membrane: Evidence for a chondroitin sulfate proteoglycan. J. Immunol., 163: 2135-2142. PMID: 10438954

Ebert, S., T. Schoeberl, Y. Walczak, K. Stoecker and T. Stempfl et al., 2008. Chondroitin sulfate disaccharide stimulates microglia to adopt a novel regulatory phenotype. J. Leukoc. Biol., 84: 736-740. DOI: $10.1189 /$ jlb.0208138

Inui, T., D. Kuchiike, K. Kubo, M. Mette and Y. Uto et al., 2013. Clinical experience of integrative cancer immunotherapy with GcMAF. Anticancer Res., 33: 2917-2919. PMID: 23780980

Inui, T., G. Katsuura, K. Kubo, D. Kuchiike and L. Chenery et al., 2016b. Case report: GcMAF treatment in a patient with multiple sclerosis. Anticancer Res., 36: 3771-3774. PMID: 27354653

Inui, T., H. Amitani, K. Kubo, D. Kuchiike and Y. Uto et al., 2016a. Case report: A non-small cell lung cancer patient treated with GcMAF, sonodynamic therapy and tumor treating fields. Anticancer Res., 36: 3767-3770. PMID: 27354652

Inui, T., K. Kubo, D. Kuchiike, Y. Uto and T. Nishikata et al., 2015. Oral colostrum macrophage-activating factor for serious infection and chronic fatigue syndrome: Three case reports. Anticancer Res., 35: 4545-4549. PMID: 26168499

Inui, T., K. Makita, H. Miura, A. Matsuda and D. Kuchiike et al., 2014. Case report: A breast cancer patient treated with GcMAF, sonodynamic therapy and hormone therapy. Anticancer Res., 34: 45894593. PMID: 25075104

Kuchiike, D., Y. Uto, H. Mukai, N. Ishiyama and C. Abe et al., 2013. Degalactosylated/desialylated human serum containing GcMAF induces macrophage phagocytic activity and in vivo antitumor activity. Anticancer Res., 33: 2881-2885. PMID: 23780974 
Masi, A., D.S. Quintana, N. Glozier, A.R. Lloyd and I.B. Hickie et al., 2015. Cytokine aberrations in autism spectrum disorder: A systematic review and meta-analysis. Mol. Psychiatry, 20: 440-446. DOI: $10.1038 / \mathrm{mp} .2014 .59$

Mohamad, S.B., H. Nagasawa, Y. Uto and H. Hori, 2002. Tumor cell alpha- $N$-acetylgalactosaminidase activity and its involvement in GcMAF-related macrophage activation. Comp. Biochem. Physiol. A Mol. Integr. Physiol., 132: 1-8.

DOI: $10.1016 / \mathrm{S} 1095-6433(01) 00522-0$

Morucci, G., J.J.V. Branca, M. Gulisano, M. Ruggiero and F. Paternostro et al., 2015. Gc-protein-derived macrophage activating factor counteracts the neuronal damage induced by oxaliplatin. Anticancer Drugs, 26: 197-209. DOI: 10.1097/CAD.0000000000000177

Németh-Csóka, M., J. Kajtár and M. Kajtár, 1975. Biological significance of helical conformation of acid polysaccharides. Connect. Tissue Res., 3: 207-211. DOI: $10.3109 / 03008207509152180$

Pacini, S., G. Morucci, T. Punzi, M. Gulisano and M. Ruggiero et al., 2012a. Effect of paricalcitol and GcMAF on angiogenesis and human peripheral blood mononuclear cell proliferation and signaling. J. Nephrol., 25: 577-581. PMID: 21956771

Pacini, S., G. Morucci, T. Punzi, M. Gulisano and M. Ruggiero, 2010. Gc protein-derived macrophageactivating factor (GcMAF) stimulates cAMP formation in human mononuclear cells and inhibits angiogenesis in chick embryo chorionallantoic membrane assay. Cancer Immunol. Immunother., 60: 479-485. DOI: 10.1007/s00262-010-0953-7

Pacini, S., T. Punzi, G. Morucci, M. Gulisano and M. Ruggiero, 2012b. Effects of vitamin D-binding protein-derived macrophage-activating factor on human breast cancer cells. Anticancer Res., 32: 45-52. PMID: 22213287

Pantazopoulos, H. and S. Berretta, 2016. In sickness and in health: Perineuronal nets and synaptic plasticity in psychiatric disorders. Neural Plast. DOI: $10.1155 / 2016 / 9847696$

Rehder, D.S., R.W. Nelson and C.R. Borges, 2009. Glycosylation status of vitamin D binding protein in cancer patients. Protein Sci., 18: 2036-2042. DOI: $10.1002 /$ pro. 214

Ruggiero, M., E. Ward, R. Smith, J.J.V. Branca and D. Noakes et al., 2014. Oleic Acid, deglycosylated vitamin D-binding protein, nitric oxide: A molecular triad made lethal to cancer. Anticancer Res., 34: 3569-3578. PMID: 24982371

Ruggiero, M., H. Reinwald and S. Pacini, 2016. Is chondroitin sulfate responsible for the biological effects attributed to the GC protein-derived Macrophage Activating Factor (GcMAF)? Med. Hypotheses, 94: 126-131.

DOI: 10.1016/j.mehy.2016.07.012
Stabler, T.V., Z. Huang, E. Montell, J. Vergés and V.B. Kraus, 2016. Chondroitin sulphate inhibits NF- $\kappa B$ activity induced by interaction of pathogenic and damage associated molecules. Osteoarthritis Cartilage. PMID: 27614315

Suh, M.J., A. Tovchigrechko, V. Thovarai, M.A. Rolfe and M.G. Torralba et al., 2015. Quantitative differences in the urinary proteome of siblings discordant for type 1 diabetes include lysosomal enzymes. J. Proteome Res., 14: 3123-3135.

DOI: $10.1021 /$ acs.jproteome.5b00052

Theoharides, T.C., I. Tsilioni, A.B. Patel and R. Doyle, 2016. Atopic diseases and inflammation of the brain in the pathogenesis of autism spectrum disorders. Transl. Psychiatry, 6: e844-e844. DOI: $10.1038 /$ tp.2016.77

Thyer, L., E. Ward, R. Smith, J.J.V. Branca and G. Morucci et al., 2013b. GC protein-derived macrophage-activating factor decreases $\alpha-\mathrm{N}$ acetylgalactosaminidase levels in advanced cancer patients. Oncoimmunology, 2: e25769-e25769. PMID: 24179708

Thyer, L., E. Ward, R. Smith, J.J.V. Branca and G. Morucci et al., 2013c. Therapeutic effects of highly purified de-glycosylated GcMAF in the immunotherapy of patients with chronic diseases. Am. J. Immunol., 9: 78-84.

DOI: 10.3844 ajisp.2013.78.84

Thyer, L., E. Ward, R. Smith, M.G. Fiore and S. Magherini et al., 2013a. A novel role for a major component of the vitamin D axis: Vitamin D binding protein-derived macrophage activating factor induces human breast cancer cell apoptosis through stimulation of macrophages. Nutrients, 5: 2577-2589. DOI: 10.3390/nu5072577

Ugarte, A., G. Bouche and L. Meheus, 2014. Inconsistencies and questionable reliability of the publication "Immunotherapy of metastatic colorectal cancer with vitamin D-binding protein-derived macrophages-activating, GcMAF" by Yamamoto et al". Cancer Immunol. Immunother., 63: 1347-1348. DOI: $10.1007 / \mathrm{s} 00262-014-1587-\mathrm{y}$

Uto, Y., T. Kawai, T. Sasaki, K. Hamada and H. Yamada et al., 2015. Degalactosylated/Desialylated bovine colostrum induces macrophage phagocytic activity independently of inflammatory cytokine production. Anticancer Res., 35: 4487-4492. PMID: 26168491

Vallières, M. and P. du Souich, 2010. Modulation of inflammation by chondroitin sulfate. Osteoarthritis Cartilage, 18: S1-S6. DOI: $10.1016 /$ j.joca.2010.02.017

Wrenshall, L.E., R.B. Stevens, F.B. Cerra and J.L. Platt, 1999. Modulation of macrophage and B cell function by glycosaminoglycans. J. Leukoc. Biol., 66: 391-400. PMID: 10496308 
Yamamoto, N. and M. Urade, 2005. Pathogenic significance of $\alpha-\mathrm{N}$-acetylgalactosaminidase activity found in the hemagglutinin of influenza virus. Microbes Infect., 7: 674-681.

DOI: 10.1016/j.micinf.2005.01.015

Yamamoto, N. and R. Kumashiro, 1993. Conversion of vitamin D3 binding protein (group-specific component) to a macrophage activating factor by the stepwise action of beta-galactosidase of B cells and sialidase of T cells. J. Immunol., 151: 2794-2802. PMID: 8360493

Yamamoto, N., 2006. Pathogenic Significance of $\alpha-\mathrm{N}-$ Acetylgalactosaminidase Activity Found in the Envelope Glycoprotein gp160 of Human Immunodeficiency Virus Type 1. AIDS Res. Hum. Retroviruses, 22: 262-271.

DOI: 10.1089 /aid.2006.22.262

Yamamoto, N., H. Suyama and N. Yamamoto, 2008. Immunotherapy for prostate cancer with Gc proteinderived macrophage-activating factor, GcMAF. Transl. Oncol., 1: 65-72. DOI: 10.1593/tlo.08106
Yamamoto, N., V.R. Naraparaju and S.M. Srinivasula, 1995. Structural modification of serum vitamin D3-binding protein and immunosuppression in AIDS patients. AIDS Res. Hum. Retroviruses, 11: 1373-1378. PMID: 8573395

Yamamoto, N., V.R. Naraparaju and S.O. Asbell, 1996. Deglycosylation of serum vitamin D3-binding protein leads to immunosuppression in cancer patients. Cancer Res., 56: 2827-2831. PMID: 8665521

Yamamoto, N., V.R. Naraparaju, M. Moore and L.H. Brent, 1997. Deglycosylation of serum vitamin $D_{3}$-binding protein by $\alpha-\mathrm{N}$-acetylgalactosaminidase detected in the plasma of patients with systemic lupus erythematosus. Clin. Immunol. Immunopathol., 82: 290-298. DOI: 10.1006/clin.1996.4320 